\title{
RETRACTED ARTICLE: SPIFGIA operators and their applications to decision making
}

\author{
Chengqing Wang ${ }^{1,2} \cdot$ Xinggan $\mathrm{Fu}^{1,2} \cdot$ Shanshan Meng ${ }^{1} \cdot{\text { Yingdong } \mathrm{He}^{1}}^{1}$
}

Received: 3 April 2016/Accepted: 19 July 2016/Published online: 3 October 2016

(C) Springer International Publishing Switzerland 2016

This article has been retracted at the request of the Editorin-Chief as it has self-plagiarized parts of other papers that had already appeared in:

1. Yingdong He, Zhen He, and Liangxing Shi, "Multiple Attributes Decision Making Based on Scaled Prioritized Intuitionistic Fuzzy Interaction Aggregation Operators”, International Journal of Fuzzy Systems, 2016, DOI: 10.1007/s40815-015-0128-y

2. Yingdong He, Zhen He, and He Huang, "Decision making with the generalized intuitionistic fuzzy power interaction averaging operators", Soft Computing, 2015, DOI: $10.1007 / \mathrm{s} 00500-015-1843-\mathrm{x}$

3. Yingdong He, Zhen He, Panpan Zhou, and Yujia Deng, "Scaled Prioritized Geometric Aggregation Operators and Their Applications to Decision Making", International Journal of Uncertainty Fuzziness and KnowledgeBased Systems, 2016. DOI: 10.1142/S0218488516500021

The online version of this article contains the full text of the retracted article as electronic supplementary material.

Electronic supplementary material The online version of this article (doi:10.1007/s41066-016-0025-2) contains supplementary material, which is available to authorized users.

Shanshan Meng

sunpeen321@gmail.com

Yingdong $\mathrm{He}$

812256307@qq.com; yidhe@tju.edu.cn

1 College of Management and Economics, Tianjin University, Tianjin 300072, China

2 Hainan College of Vocation and Technique, Haikou 570216, China 